\title{
Health-related quality of life in metabolically healthy obese individuals
}

\section{Korhonen, Paivi E.}

2017

Korhonen , P E \& Kautiainen , H 2017 , ' Health-related quality of life in metabolically healthy obese individuals ', Obesity Research \& Clinical Practice, vol. 11 , no. 4 , pp. 499-500 . https://doi.org/10.1016/j.orc

http://hdl.handle.net/10138/297918

https://doi.org/10.1016/j.orcp.2017.06.005

publishedVersion

Downloaded from Helda, University of Helsinki institutional repository.

This is an electronic reprint of the original article.

This reprint may differ from the original in pagination and typographic detail.

Please cite the original version. 


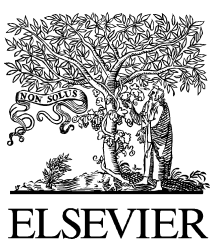

RESEARCH LETTER

Health-related quality of life in metabolically healthy obese individuals

\section{Introduction}

Metabolically healthy obese (MHO) individuals have low burden of cardiometabolic risk factors. We aimed at investigating whether $\mathrm{MHO}$ individuals also have better health-related quality of life (HRQoL) than metabolically abnormal obese (MAO) individuals in a Finnish apparently healthy, middleaged population.

\section{Material and methods}

A population survey was carried out in an industrialised Finnish small town Harjavalta in 2005-2006. We posted a risk factor survey and a tape for measurement of waist to all home-dwelling persons aged $45-70$ years $(n=2856)$. Participation rate was $73 \%$. All respondents with waist circumference $\geq 80 \mathrm{~cm}$ in women and $\geq 94 \mathrm{~cm}$ in men were invited for further examination.

Public health nurses examined 1469 subjects. Body mass index (BMI) was calculated as weight $(\mathrm{kg})$ divided by the square of height $\left(\mathrm{m}^{2}\right)$. Blood pressure was measured twice, and the mean value was used in the analysis. Glucose and lipid profile were determined in fasting plasma samples. HRQoL was assessed with the Short-form Health Survey (SF-36) [1] before the physical examination was performed. Metabolic syndrome was defined according to the harmonisation definition [2]. MHO and MAO phenotypes were defined as $\mathrm{BMI} \geq 30.0 \mathrm{~kg} / \mathrm{m}^{2}$ without or with metabolic syndrome, respectively. Statistical comparisons between groups were made by analysis of variance or chi square test. Information about chronic comorbidities and medication was gathered from questionnaires and medical records. For the analyses described here, patients with any chronic comorbidities were excluded.

\section{Results}

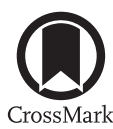

Of the 1187 study subjects without chronic comorbidities, 319 (26.9\%) were obese (mean age $57.4 \pm 6.8$ years, $60.8 \%$ women). The prevalence of MHO phenotype among obese individuals was $31.0 \%$ $(99 / 319)$.

Table 1 shows the characteristics and the HRQoL dimensions of the subjects according to obesity phenotypes. Although the general health scale was impaired in MAO subjects, the mental and physical component summaries were comparable between $\mathrm{MHO}$ and $\mathrm{MAO}$ individuals.

\section{Discussion}

We conclude that in Finnish obese subjects without co-morbidities, the overall $\mathrm{HRQOL}$ is equal in $\mathrm{MHO}$ and MAO individuals. Of the SF-36 dimensions, only general health scale was better among MHO than MAO subjects.

For the time being, there is no standardised definition to identify MHO individuals for research protocols or in clinical practice. We used the same definition as the Finnish national survey, in which MHO phenotype was observed in $9 \%$ of obese men and in $16 \%$ of obese women aged $45-74$ years [3]. In our cohort, the MHO phenotype was more frequent probably due to younger and apparently healthy participants. The prevalence of obesity in Finnish adult population is estimated to be $21 \%$ in women and $19 \%$ in men [4].

Also differences in study sample populations and methods used make direct comparisons between studies difficult. The same definition of MHO and the same HRQoL measure as in the present study were used in an Australian study among randomly selected adults $\geq 18$ years old and free of cardiovascular disease [5]. In this study population, the MHO demonstrated higher mental and physical summary scores than MAO subjects, although the exact figures were not reported [5]. Usually, a 3-5-point 
Table 1 Characteristics and health-related quality of life dimensions of the obese individuals according to metabolic status.

\begin{tabular}{llll}
\hline & $\begin{array}{l}\text { MHO } \\
\mathrm{n}=99\end{array}$ & $\begin{array}{l}\text { MAO } \\
\mathrm{n}=220\end{array}$ & P-Value \\
\hline Demographics & & & \\
Age, years, mean (SD) & $56.6(6.4)$ & $57.8(6.9)$ & 0.12 \\
Female, $\mathrm{n}(\%)$ & $68(68.7)$ & $126(57.3)$ & 0.053 \\
White collar worker, $\mathrm{n}(\%)$ & $29(30.2)$ & $53(25.0)$ & 0.34 \\
Current smoker, $\mathrm{n}(\%)$ & $16(16.2)$ & $34(15.5)$ & 0.87 \\
Body mass index, kg/m², mean (SD) & $33.4(4.0)$ & $34.1(4.0)$ & 0.17 \\
SF-36, mean (SD) & & & \\
Physical functioning & $79.5(20.9)$ & $76.2(21.3)$ & 0.20 \\
Role physical & $76.3(34.1)$ & $73.9(37.2)$ & 0.59 \\
Bodily pain & $68.8(23.7)$ & $69.0(24.2)$ & 0.95 \\
General health & $62.4(17.1)$ & $57.5(17.7)$ & 0.023 \\
Vitality & $69.4(19.6)$ & $66.7(20.1)$ & 0.29 \\
Social functioning & $87.5(19.4)$ & $87.6(18.1)$ & 0.98 \\
Role emotional & $87.8(26.0)$ & $84.1(30.1)$ & 0.30 \\
Mental health & $80.2(15.3)$ & $77.9(17.1)$ & 0.26 \\
Physical component summary & $46.1(8.8)$ & $44.8(9.5)$ & 0.28 \\
Mental component summary & $54.6(8.6)$ & $53.7(8.8)$ & 0.39 \\
\hline
\end{tabular}

Abbreviations: $\mathrm{MHO}$, metabolically healthy obese; MAO, metabolically abnormal obese; SF-36, Short-form Health Survey.

difference in the SF-36 scores is considered clinically relevant $[1,6]$.

The major limitation of our study is the crosssectional design, so we cannot determine any causal relationships. The strengths of the study are that the data comes from a community-based sample of the middle-aged Finnish population, chronic illnesses affecting HRQoL were excluded, and the measurements were made by trained medical staff.

\section{References}

[1] Hays RD, Morales LS. The SF-36 measure of health-related quality of life. Ann Med 2001;33:350-7.

[2] Alberti KG, Eckel RH, Grundy SM, Zimmet PZ, Cleeman JI, Donato KA, et al. Harmonizing the metabolic syndrome. A joint interim statement of the International Diabetes Federation task force on epidemiology and prevention; National Heart, Lung, and Blood Institute; American Heart Association; World Heart Federation; International Atherosclerosis Society; and International Association for the Study of Obesity. Circulation 2009;120:1640-5.

[3] Pajunen P, Kotronen A, Korpi-Hyövälti E, KeinänenKiukaanniemi S, Oksa H, Niskanen L, et al. Metabolically healthy and unhealthy obesity phenotypes in the general. population: the FIN-D2D Survey. BMC Public Health 2011;11:754
[4] Peltonen M, Harald K, Männistö S, Saarikoski L, Peltomäki P, Lund L, et al. The National FINRISK 2007 Study. Publications of the National Public Health Institute, B34/2008.

[5] Appleton SL, Seaborn CJ, Visvanathan R, Hill CL, Gill TK, Taylor AW, et al. Diabetes and cardiovascular disease outcomes in the metabolically healthy obese phenotype. Diabetes Care 2013;36:2388-94.

[6] Wyrwich KW, Tierney WM, Babu AN, Kroenke K, Wolinsky FD. A comparison of clinically important differences in healthrelated quality of life for patients with chronic lung disease, asthma, or heart disease. Health Serv Res 2005;40:577-91.

Päivi E. Korhonen* Department of General Practice, Turku University and Turku University Hospital, Turku, Finland

Hannu Kautiainen ${ }^{\mathrm{a}, \mathrm{b}}$

a Unit of Primary Health Care, Helsinki University Central Hospital, 00029 Helsinki, Finland ${ }^{\mathrm{b}}$ Department of General Practice, University of Helsinki, 00014 Helsinki, Finland

* Corresponding author at: Jokikatu 3, 29200 Harjavalta, Finland. Fax: +358 26741180. E-mail address: paikor@utu.fi (P.E. Korhonen)

27 January 2017 